\title{
Echocardiographic features of Candida species endocarditis: 12 cases and a review of published reports
}

\author{
E Donal, P Abgueguen, D Coisne, J P Gouello, E P McFadden, J Allal, P Corbi
}

\begin{abstract}
Objective-To describe the specific echocardiographic features of Candida species endocarditis. Design-Retrospective review of the case records of patients with confirmed candida endocarditis.

Setting-Cases referred to three French university centres over an eight year period were studied.

Design-12 patients with confirmed Candida species endocarditis infection were identified. The transthoracic $(n=12)$ and transoesophageal $(n=12)$ echocardiographic appearances were compared with the surgical findings $(n=10)$.

Results-Large dense heterogeneous vegetations were found in 11/12 cases. A hyperechogenic heterogeneous myocardial texture, observed in seven of the 12 patients, was associated with extensive myocardial damage at surgery. While it was possible to diagnose candidal cardiac infection in all patients by transthoracic echocardiography, transoesophageal echocardiography was useful for optimal assessment of the valvar and paravalvar structures.

Conclusions-In the setting of endocarditis, the detection of myocardial involvement, which is characterised by a heterogeneous myocardial texture, is an argument in favour of Candida species endocarditis and may warrant early surgical intervention.

(Heart 2001;86:179-182)
\end{abstract}

Keywords: echocardiography; Candida species; endocarditis

Echocardiography is an extremely effective tool for the diagnosis of endocarditis. Candida species endocarditis is a rare form of the condition, in which coexisting myocardial involvement is not uncommon. It has a particularly poor prognosis. In this report we present 12 new cases of cardiac infection with Candida species. We describe the unusual appearances of vegetations in this particular form of endocarditis and report abnormalities in the texture of the myocardium on echocardiography-shown at surgery to represent myocardial involvement-which we feel have important implications when planning surgical intervention.

In this rare condition, echocardiography is helpful both in making a diagnosis and in guiding the treatment strategy. Some investigators consider that the size of the vegetations is of paramount importance in the decision to undertake surgical intervention. ${ }^{1}$ While this is a major factor, we provide evidence suggesting that the echocardiographic texture of the infected cardiac tissue is also important and may be an argument in favour of early surgical intervention.

\section{Methods}

PATIENTS

From the records of the echocardiography departments of three French University Hospitals (Hôpital Bichat-Paris, Angers, and Poitiers), we identified 12 patients, who, between October 1991 and September 1999, underwent echocardiography for suspected endocarditis and subsequently had Candida species endocarditis confirmed, either on repeated blood cultures (10 cases) or on culture of valve tissue removed at surgery (two cases).

The mean age of the patients was 41 years (range 21-81 years). Eight were men. Four were intravenous drug users, two of whom were HIV positive. Two patients had mechanical cardiac valves, one a permanent cardiac pacemaker, and one an indwelling venous catheter. All patients were classified in the category of definite endocarditis according to the Duke criteria. ${ }^{2}$

\section{ECHOCARDIOGRAPHY}

All 12 patients underwent both transthoracic and transoesophageal echocardiography, the diagnosis of endocarditis being made exclusively on the transthoracic examination (table 1). Transoesophageal echocardiography was carried out in order to obtain more detailed information on the size and location of the vegetations, the degree of involvement of valvar or paravalvar structures, the presence of abscess formation, and so on.

\section{Results}

ECHOCARDIOGRAPHIC FINDINGS

Left ventricular function was normal in all patients. Two had small pericardial effusions. Aortic valve involvement was present in six patients, mitral valve involvement in three, and tricuspid valve involvement in three; multiple valves were affected in two patients. Of the three patients with tricuspid endocarditis, one was an intravenous drug user, one had an infected pacemaker (infected thrombus in the 


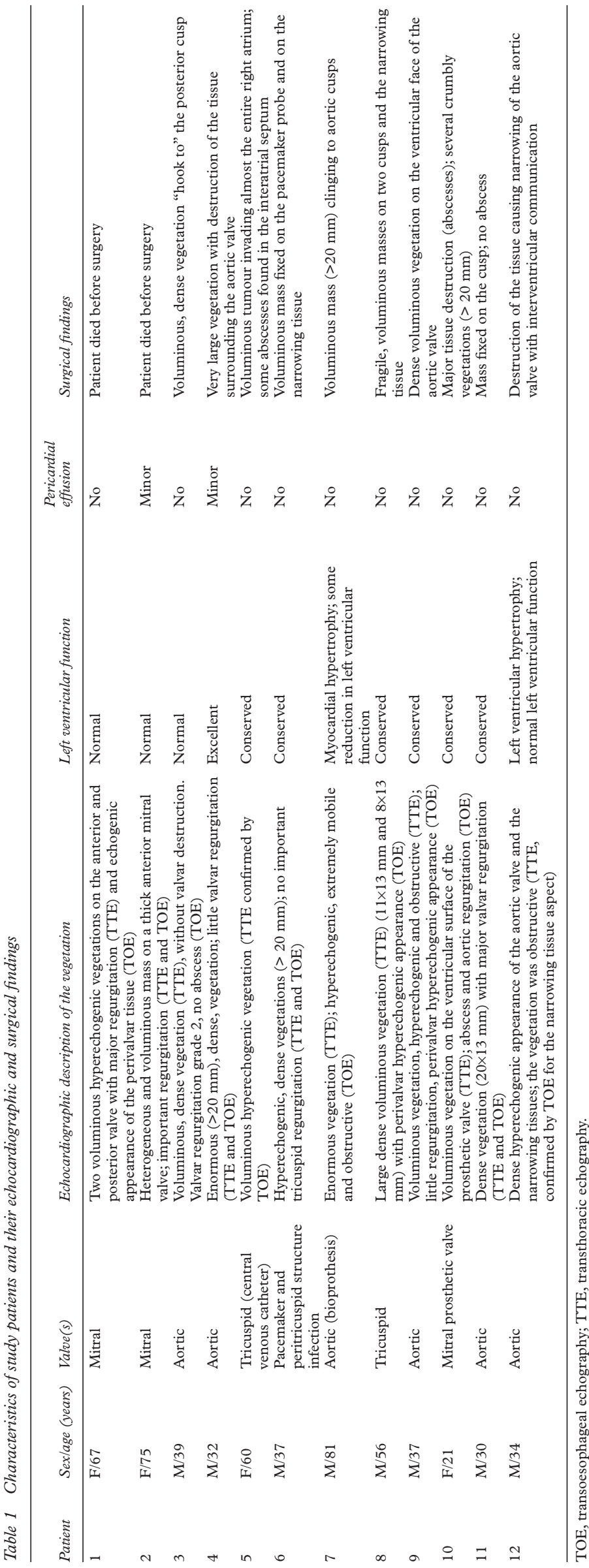

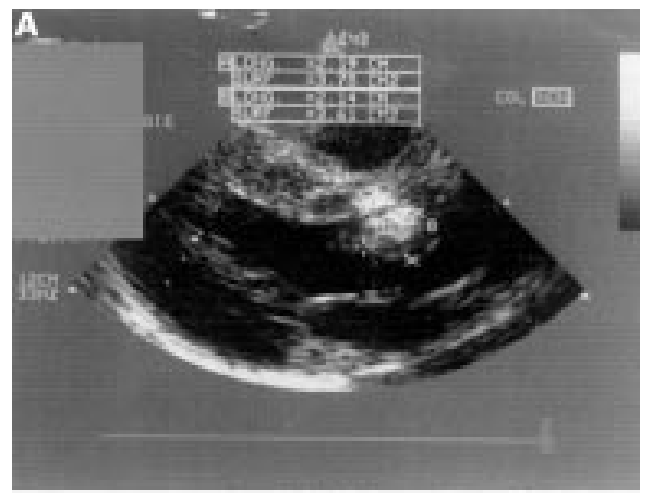

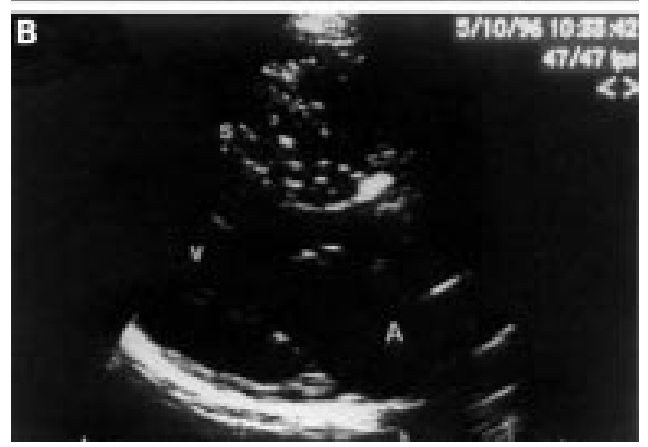

Figure 1 Echocardiographic example of Candida species aortic valvar endocarditis, with destruction of the

narrowing tissue. There was initially a bulky, echogenic mass on the aortic cusps (A). Rapidly, the

echocardiographic aspect of the interventricular septum became unusual with a large number of hyperechogenic points. Anatomical observation revealed a large number of micro-abscesses of the narrowing of the aortic valve(B). $S$, interventricular septum; $V$, left ventricular cavity; $A$, left atrium.

right atrium without tricuspid valve destruction), and the third had an infected indwelling central venous catheter.

In seven of the 12 patients, the vegetations were described as being abnormally large (fig 1). The size of each vegetation was not precisely described on all the echocardiography reports. The mean size (in four patients) was $20 \mathrm{~mm}$ (range 11-32 $\mathrm{mm}$ ). Vegetations were obstructive in two cases. One patient with aortic endocarditis had a heterogeneous, hyperechogenic appearance of the interventricular septum (fig 1). Echocardiographic appearances suggestive of the presence of an abscess were seen in three cases. In one patient, there appeared to be an aneurysm within an aortic cusp. A frequently used phrase (seven cases) in the echocardiography reports describing the echocardiographic appearances of the vegetations was "a voluminous mass adhering to the valvar or paravalvar structures". In 11 of the 12 patients, the adjectives "dense" and "hyperechogenic" were used (fig 1). In all cases, there was a notable discrepancy between the large size of the vegetation and the rather mild grade of the associated valve regurgitation (fig 2).

\section{SURGICAL FINDINGS}

Surgical findings were available in 10 patients. Two patients died rapidly before surgical intervention. All patients were admitted to an intensive care unit because of severe septic shock. Complete destruction of the valve and the surrounding tissue was observed in six 


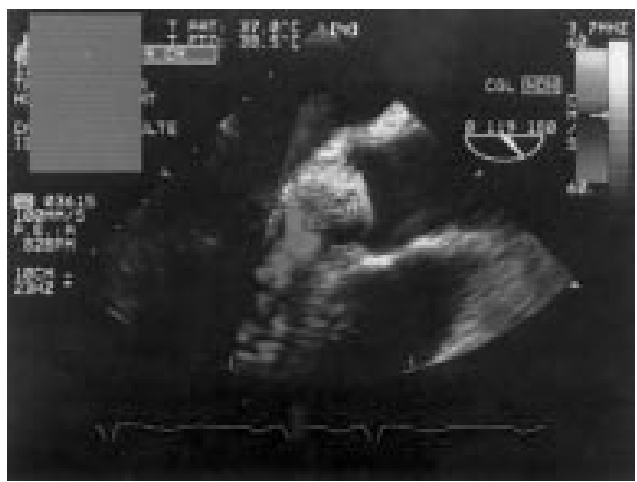

Figure 2 Transoesophageal view of an aortic valve fungal endocarditis, with voluminous, hyperechogenic vegetation and moderate valvar regurgitation.

cases. In seven cases, the anatomical description of the vegetation indicated the presence of an infiltrated and retracted appearance of the infected cardiac valve. In two cases, the perioperative description of the vegetation was similar to that of bacterial endocarditis. The size of the vegetation was never precisely described by the surgeon because of the extent of fungal lesions.

There was a good correlation between the echocardiographic and surgical findings in all but two cases. In one of these, echocardiography overestimated the extent of tissue destruction. In the other, a heterogeneous, hyperechogenic appearance of the interventricular septum was noted on echocardiography.

At surgery, multiple interventricular communications were found. Surgery was successful in all patients. A new model of biological valve was used in the last three patients in the series. This was a stentless valve capable of replacing the damaged valves and also the surrounding tissues. It had a stentless soft root which could be used as a patch, permitting the surgeon, for example, to close the interventricular communication in one of the 12 patients.

Seven patients were discharged alive. All seven were still alive after two years of follow up. Three patients died in the 15 days following the surgical treatment: two of these died suddenly, while the third patient died in septic shock.

\section{Discussion}

Cardiac infections by Candida species are rare but have usually had a dismal prognosis. Vo and colleagues reported an $80 \%$ mortality rate in a series of 44 patients described in various published reports. ${ }^{3}$ Several investigators have suggested that the definitive eradication of fungal endocarditis may not be possible, based on the observed frequency of late recurrence. Branco and associates have emphasised the importance of early surgical intervention in view of the high rate of embolic events, often in multiple locations, and the frequent occurrence of perivalvar destruction leading to heart failure. $^{4}$

Endocarditis is probably not the most common cardiac location of fungal infection: in a necropsy series of 51 patients, Walsh and colleagues found 31 cases of myocarditis, 13 of endocarditis, and four of pericarditis; in three cases all three locations were involved. ${ }^{5}$ Myocarditis was generally asymptomatic and was statistically linked to the presence of HIV infection. The two HIV patients in our series had voluminous and extensive masses adherent to the endocardium but neither had diffuse myocarditis. Numerous yellowish grainy spots, with a surrounding haemorrhagic halo, were observed throughout the myocardium. Similar appearances were described by Andriole and colleagues in a series of 21 necropsied cases of fungal endocarditis. ${ }^{6}$ In $38 \%$ of cases of candida endocarditis from published necropsy series, symptoms began soon after thoracic surgery.

Other clinical settings are associated with fungal endocarditis. In a report by Lozano and associates, $50 \%$ of patient with fungal endocarditis were drug addicts and $60 \%$ had preexisting valvar disease. ${ }^{7}$ Branco and colleagues reported a series of 10 patients with fungal endocarditis, in eight of whom the infection developed on a prosthetic valve; none of these patients was an intravenous drug user. ${ }^{4}$

In the present series, four of the 12 patients were intravenous drug users and a further four patients had either a prosthetic valve, a pacemaker, or an indwelling central venous catheter. Pre-existing valvar disease was only seen in patients with left sided infection.

Fungal valvar infection is aggressive and difficult to treat surgically. There is a high risk of embolisation, and serious perioperative bleeding may occur when infected tissue is resected. ${ }^{8}$ Usually surgery is complicated by the need for radical debridement and reconstruction of the valvar root. Some investigators have suggested that immediate surgical intervention is associated with a better long term outcome. ${ }^{9}$ Fungal prosthetic valve endocarditis is particularly serious, and the first episode is usually a result of nosocomial candidaemia. ${ }^{10}$ Recurrent episodes are frequent. ${ }^{11}$ In prosthetic valve fungal endocarditis, urgent surgical intervention is recommended and antifungal drug treatment should be continued for life.

Echocardiography has become a mainstay in the diagnosis of endocarditis. Transthoracic echocardiography alone is often sufficient to establish the diagnosis, as demonstrated by Lengyel, who found that transthoracic and transoesophageal echocardiographic findings were concordant in $83 \%$ of cases. ${ }^{12}$ However, transoesophageal echocardiography is superior for defining the anatomical extent of the valvar lesions and guiding the surgical strategy. ${ }^{12}$ In the subgroup of patients with suspected endocarditis on prosthetic valves, transoesophageal echocardiography is preferable. ${ }^{13}$ In addition, Erbel and colleagues have shown that transoesophageal echocardiography can identify a high risk subgroup among patients with infective endocarditis. ${ }^{14}$ Nguyen and associates drew attention to the diagnostic value of transoesophageal echocardiography in candidal prosthetic valve endocarditis, but did not report on specific echocardiographic features of the vegetations or the myocardium. ${ }^{15}$ Other case reports have occasionally described large 
vegetations on transthoracic echocardiography. ${ }^{16}{ }^{17}$ Tischler and Vaitkus published a meta-analysis of the findings at echocardiography. ${ }^{1}$ They found that vegetation size was $<10 \mathrm{~mm}$ in approximately $50 \%$ of cases of bacterial endocarditis. In our study, the diagnosis was made on transthoracic examination in all patients. Large vegetations - around $20 \mathrm{~mm}$ in diameter-were common. These were usually hyperechogenic and dense. Transoesophageal examination was helpful in ascertaining the extent of particularly large vegetations, in detecting abscesses, and in looking for other evidence of tissue destruction. In three cases in our series (a man of 32, a man of 34, and a woman of 60), echocardiography underestimated the extent of disease as determined at surgery. This may have been related to the delay between echocardiography and surgical intervention. In our opinion it is important to highlight the hyperechogenic appearances of valvar or perivalvar lesions. These are particularly characteristic of candidal infection.

\section{CONCLUSIONS}

Candidal cardiac infection is a rare condition with a poor prognosis. Our study emphasises the unusual echocardiographic features of endocarditis caused by Candida species. Transthoracic echocardiograpy of the vegetations is striking because of their heterogeneous and dense appearance. These characteristic appearances should suggest the diagnosis of candida rather than other forms of endocarditis and hence influence the treatment strategy. When this dense heterogeneous appearance is found not only in the vegetations but also in the adjacent myocardium, early surgical intervention may be warranted.
We thank Eugene McFadden for his assistance and suggestions in preparing the manuscript.

1 Tischler MD, Vaitkus PT. The ability of vegetation size on echocardiography to predict clinical complications: a metaechocardiography to predict clinical complication

2 Hoen B, Beguinot I, Rabaud C, et al. The Duke criteria for diagnosing infective endocarditis are specific: analysis of 100 patients with acute fever or fever of unknown origin. Clin Infect Dis 1996;23:298-302.

3 Vo NM, Russell JC, Becker DR. Mycotic emboli of the peripheral vessels: analysis of forty-four cases. Surgery 1981;90:541-5.

4 Branco L, Pitta ML, Bernardes L, et al. A review of infectious endocarditis due to Candida. Rev Port Cardiol 1997;16:967-4

5 Walsh TJ, Hutchins GM, Bulkley BH, et al. Fungal infections of the heart: analysis of 51 autopsy cases. $A m \mathcal{F}$ Cardiol 1980;45:357-66.

6 Andriole VT, Kravetz HM, Roberts WC, et al. Candida endocarditis. Am f Med 1962;32:251-85.

7 Lozano P, Flores D, Blanes I, et al. Acute lower limb ischemia complicating endocarditis due to Candida parapsilosis in a drug abuser. Ann Chir Vasc 1994;8:591-4.

8 Remsey ES, Lytle BW. Repair of fungal aortic prosthetic valve endocarditis associated with periannular abscess. $\mathcal{F}$ Heart Valve Dis 1998;7:235-9.

9 Hogevik H, Alestig K. Fungal endocarditis. A report on seven cases and brief review. Infection 1996;24:17-21.

10 Muehrcke DD, Lytle BW, Cosgrove DM. Surgical and long-term antifungal therapy for fungal prosthetic valve endocarditis. Ann Thorac Surg 1995;60:538-43.

11 Nasser RM, Melgar GR, Longworth DL, et al. Incidence and risk of developing fungal prosthetic valve endocarditis after nosocomial candidemia. Am F Med 1997;103:25-32.

12 Lengyel $M$. The impact of transesophageal echocardiography on the management of prosthetic valve endocarditis: experience of 31 cases and review of the literature. $\mathcal{F}$ Heart Valve Dis 1997;6:204-11.

13 Irani WN, Grayburn PA, Afridi I. A negative transthoracic echocardiogram obviates the need for transesophageal echocardiography in patients with suspected native valve echocardiography in patients with suspected native
infective endocarditis. Am $\mathcal{7}$ Cardiol 1996;78:101-3.

14 Erbel R, Liu F, Ge J, et al. Identification of high-risk subgroups in infective endocarditis and the role of echocardiography. Eur Heart f 1995;16:588-602.

15 Nguyen MH, Nguyen ML, Yu VL, et al. Candida prosthetic valve endocarditis: prospective study of six cases and review of the literature. Clin Infect Dis 1996;22:262-7.

16 Inoue $\mathrm{Y}, \mathrm{Yozu} \mathrm{R}, \mathrm{Ueda} \mathrm{T}$, et al. A case report of Candida parapsilosis endocarditis. F Heart Valve Dis 1998;7:240-2.

17 Gilbert HM, Peters ED, Lang SJ, et al. Successful treatment of fungal prosthetic valve endocarditis report and review. Clin Infect Dis 1996;22:348-54.

\section{1st Asia Pacific Forum on Quality Improvement in Health Care}

\section{Three day conference}

\section{Wednesday 19 to Friday 21 September 2001 Sydney, Australia}

We are delighted to announce this forthcoming conference in Sydney. Authors are invited to submit papers (call for papers closes on Friday 6 April), and delegate enquiries are welcome.

The themes of the Forum are:

- Improving patient safety

- Leadership for improvement

- Consumers driving change

- Building capacity for change: measurement, education and human resources

- The context: incentives and barriers for change

- Improving health systems

- The evidence and scientific basis for quality improvement.

Presented to you by the BMJ Publishing Group (London, UK) and Institute for Healthcare Improvement (Boston, USA), with the support of the the Commonwealth Department of Health and Aged Care (Australia), Safety and Quality Council (Australia), NSW Health (Australia), and Ministry of Health (New Zealand).

For more information contact: quality@bma.org.uk or fax +44(0)20 73836869 